\title{
Systematic Review of the profile of emergency contraception users
}

\author{
Maria de Lluc Bauzà Amengual ${ }^{1}$ \\ Magdalena Esteva Canto² \\ Inmaculada Pereiro Berenguer ${ }^{3}$ \\ Maria Ingla Pol ${ }^{4}$
}

\begin{abstract}
Objective: to discern the profile of the Spanish Emergency Contraceptive users (EC). Design: systematic review of contraceptive use in the Spanish population. Data Source: Spanish and international databases, between January 2006 - March 2011. Keywords: Contraceptives, Postcoital pills, emergency contraception, levonorgestrel, data collection. Study selection: original papers, letters to the editor in which stated aims were the description, prediction or measurement of variables related to EC use. Twenty-two papers were retrieved and fourteen were finally selected, all of which were descriptive. Data extraction: manuscripts were evaluated by two independent reviewers. Results: Women requesting EC have ages between 21-24 years, mostly single and university students; declare that they have not previously used EC, and attend an Emergency department, at weekends and within 48 hours following unprotected sexual intercourse. The reason is condom rupture. None of the studies reviewed measured alcohol and other drug consumption, the number of sexual partners, nor any of the studies performed a comparison with a group not using EC. Conclusions: lack of homogeneity and comprehensiveness of studied variables resulted in a limited profile of Spanish EC users. Further studies are needed with a more comprehensive approach if sexual health interventions are to be carried out in possible users.
\end{abstract}

Descriptors: Contraception, Postcoital; Data Collection; Levonorgestrel.

\footnotetext{
${ }^{1}$ MSc, Full Professor, Clinical Assistant Professor, School of Nursing and Physiotherapy, University of Balearic Islands, Mallorca, Spain.

2 PhD, Researcher, Research Unit, Majorca Primary Care Department, Balearic Health Service, Majorca, Spain.

${ }^{3}$ Doctor, Health center primary care Puerto de Sagunto II, Valencia, Spain.

${ }^{4}$ Nurse, Health and consumer care department, Palma City Council, Mallorca, Spain.
}

Bauzà-Amengual ML, Esteva-Cantó M, Pereiro-Berenguer I, Ingla-Pol M. Systematic Review of the profile of emergency contraception users. Rev. Latino-Am. Enfermagem. 2016;24:e2733. [Access ___ _ _ ] ; Available in: DOI: http://dx.doi.org/10.1590/1518-8345.0882.2733 month day year 


\section{Introduction}

Throughout the world, unplanned pregnancy is a considerable social and public health issue. The majority of cases are unwanted pregnancies and many of these cases result in abortion. Pregnancies in girls between 14 and 17 years rose from 4\%o in 1990 to almost $12 \%$ in $2006^{(1)}$ This may have been due to two factors. Firstly, that contraception is either not used or is used incorrectly in many cases. Secondly, that penetrative sexual relations are being initiated at a younger age; and the younger age leads to a decrease in risk perception ${ }^{(2)}$

The rate of abortion or voluntary pregnancy termination (VPT) represents an estimate of the number of unwanted pregnancies. On analysing the incidence of this rate in Spain, we see moderate values with respect to other European countries (3). It should be pointed out that Spain is one of the countries that have seen the greatest increase in recourse to abortion in recent decades, rising from 7.14 VPT per 1,000 women in the year 2000 , to 11.41 in 2009 . The abortions are mainly in the 20-29 age group, and among those who have sophomore-10h-grade ${ }^{(4)}$.

In the second half of the $20^{\text {th }}$ century, the emergence of contraception and its massive use in the developed world represented an effective measure for birth control and the avoidance of unwanted pregnancies. Lately, scientific advances have led to other types of drugs entering the market, known as emergency contraception (EC) or the 'post-coital pill'. EC use can be defined as taking a contraceptive drug (also known as the 'morningafter pill') up to 72 hours after unprotected coitus with the aim of preventing an unwanted pregnancy. Its mechanism of action is to impede ovulation or fertilisation, but once the fertilized egg is implanted the pill will not have any effect ${ }^{(5)}$. The conclusions of clinical trials on the efficacy of these drugs support the use of Progestogen only (total dose of $1.5 \mathrm{mg}$ of levonorgestrel) as the method of emergency contraception of choice due to greater effectiveness and lower incidence of nausea and vomiting (6). The introduction of these drugs to the market considerably raised expectations regarding prevention and reduction of the number of VPT and it has even been argued that its use could prevent up to $95 \%$ of unwanted pregnancies (7).

On March 23rd, 2001, the government in Spain authorised the sale of levonorgestrel as EC with a medical prescription. Since then, the various Spanish regions legalised its provision. This confirms that no common protocol exists in the National Health Service (NHS) establishing the conditions for the supply of EC among regional health services. The inclusion of EC in the catalogue of contraceptives has involved numerous debates and controversies. In 2003, 317,670 morningafter pills were dispensed, which corresponds to $3 \%$ of women of childbearing age, indicating a sharp increase in consumption ${ }^{(8)}$. From September 2009, the so-called 'morning-after pill' could be acquired without a medical prescription in Spanish pharmacies. This measure aims to facilitate access to this pill for all women who require it, at the appropriate time to ensure its efficacy, irrespective of their place of residence and the regional laws in effect.

This deregulation of the pill and improvements in access to it, help to overcome certain obstacles for women such as the shame they may feel in consulting a health professional, as well as facilitating its anonymous purchase at pharmacies. From another point of view, such a high level of use could be interpreted as a failure, as access to contraception has not prevented the increase in abortions, nor has the availability of condoms reduced demands for the morning-after pill. Rather, it seems that these are factors which, taken together, may encourage people to enter into risky situations or remain in them ${ }^{(9)}$. Thus, the improvements in EC accessibility have led to the formulation of three more pragmatic concerns (10): 1) Whether easy access to the EC pill increases early sexual activity, 2) Whether women using this method repeatedly tend to abandon their habitual contraceptives; and 3) Whether these factors expose women and their partners to a greater risk of sexually transmitted diseases.

Following these legislative changes, there is a need to analyse the current situation of women seeking EC in our country. Hence, the aim of this study is to discern the profile of EC users in Spain through a systematic review of the literature so that strategies can subsequently be designed to address the population of actual female users.

\section{Method}

A systematic review of the literature published on emergency contraception use in the Spanish population was performed with the aim to discern the profile of the Spanish Emergency Contraceptive users. The bibliographic search was carried out in the main Spanish and international databases: PubMed, Cuidenplus, BDIE, 
CINAHL, EMBASE, Cochraneplus, ExcelenciaClínica, Joanna Briggs, IBECS, IME, OLID, ISOC and Ageline. The key words used were: Contraceptives, Postcoital pills, emergency contraception, levonorgestrel, data collection. Boolean operators and classical truncations were used (OR, AND, *).

Search inclusion criteria were as follows: original articles and letters to the editor published during the last five years (2006 to March, 2011) in English, Spanish or French, and directly or indirectly focused on the description, prediction or measurement of variables related to the use of emergency contraception in Spain.

A manual search was also performed using the references in the articles and reviews retrieved to identify those articles that had not been captured in the electronic search. Thus we were able to find articles published in Spanish journals that were not indexed in the above databases but had been subject of a peerreview process. Then, by reading the title and abstract, the papers meeting the inclusion criteria were selected. The full texts of the manuscripts selected were obtained so that pairs of researchers could subsequently assess them independently and analyse them through a grid designed for this study. To design the analytical grid, meetings were held within the research team until consensus was reached on the definitive analysis grid. Two reviewers retrieved data independently. Information was collected on the study design, aims, scope, participants, sample size, variables related to the use of $\mathrm{EC}$, main results and conclusions. The variables gathered were socio-demographic variables, variables related to the use of EC (frequency, time elapsed since coitus, reason for use, prescription services) and variables connected with sexual habits (age at initiation of sexual relations, obstetrics history). The measures used are those presented in the studies, mainly percentages and means with standard deviations. The studies were evaluated separately and a general assessment was done to ensure they met minimum quality standards. The bibliographic reviews were conducted during the month of May of 2010 and during March of 2011.

\section{Results}

Twenty-two articles were retrieved and fourteen were finally selected, the majority $(92.8 \%)$ in the Spanish language. Figure 1 shows the characteristics of the published articles (11-24). The studies selected were carried out between 1999 and 2008 and represent nine Spanish regions; all the studies had a descriptive crosssectional design; no qualitative studies were found. In eight studies the data were collected from emergency services and the majority focused on women who requested EC, without a comparison group. Only two included both genders as the subjects of the studies dealt with adolescents and college students. Ages ranged from 13-53 years across the selected studies.

\begin{tabular}{|c|c|c|c|c|c|c|c|c|}
\hline $\begin{array}{c}\text { Year of } \\
\text { publication }\end{array}$ & First author & Place & $\begin{array}{l}\text { Date data } \\
\text { collection }\end{array}$ & $\begin{array}{l}\text { Inclusion of } \\
\text { cases }\end{array}$ & $\begin{array}{c}\text { Study } \\
\text { Subjects }\end{array}$ & Source of data & $\begin{array}{l}\text { Sample } \\
\text { Size }\end{array}$ & $\begin{array}{l}\text { Age } \\
\text { range }\end{array}$ \\
\hline 2002 & $\begin{array}{c}\text { Aginaga JR } \\
\quad(13)\end{array}$ & Guipúzcoa & 2001 & $\begin{array}{l}\text { Primary care } \\
\text { emergency } \\
\text { departments }\end{array}$ & $\begin{array}{l}\text { Women who } \\
\text { request it }\end{array}$ & $\begin{array}{c}\text { Ad hoc } \\
\text { Questionnaire }\end{array}$ & $\begin{array}{c}163 \\
\text { women }\end{array}$ & $14-43$ \\
\hline 2002 & $\begin{array}{c}\text { Cárdenas DP } \\
\text { (14) }\end{array}$ & Almería & 2001-2002 & $\begin{array}{l}\text { Hospital } \\
\text { emergency } \\
\text { rooms and } \\
\text { primary care } \\
\text { emergency } \\
\text { departments }\end{array}$ & $\begin{array}{l}\text { Women who } \\
\text { request it }\end{array}$ & $\begin{array}{c}\text { Ad hoc } \\
\text { Questionnaire }\end{array}$ & $\begin{array}{c}446 \\
\text { women }\end{array}$ & - \\
\hline 2002 & Ruíz S (15) & Madrid & 2000 & $\begin{array}{l}\text { Family planning } \\
\text { centres }\end{array}$ & $\begin{array}{l}\text { Women who } \\
\text { request it }\end{array}$ & $\begin{array}{c}\text { Ad hoc } \\
\text { Questionnaire }\end{array}$ & $\begin{array}{c}404 \\
\text { women }\end{array}$ & $14-49$ \\
\hline
\end{tabular}


figura 1 - (continuation)

\begin{tabular}{|c|c|c|c|c|c|c|c|c|}
\hline $\begin{array}{c}\text { Year of } \\
\text { publication }\end{array}$ & First author & Place & $\begin{array}{l}\text { Date data } \\
\text { collection }\end{array}$ & $\begin{array}{c}\text { Inclusion of } \\
\text { cases }\end{array}$ & $\begin{array}{c}\text { Study } \\
\text { Subjects }\end{array}$ & Source of data & $\begin{array}{l}\text { Sample } \\
\text { Size }\end{array}$ & $\begin{array}{l}\text { Age } \\
\text { range }\end{array}$ \\
\hline 2003 & Lete I (16) & Spain & 2002 & $\begin{array}{c}\text { Primary care } \\
\text { centres, primary } \\
\text { care emergency } \\
\text { departments, } \\
\text { family planning } \\
\text { centres, } \\
\text { hospital } \\
\text { emergency } \\
\text { rooms and } \\
\text { gynecology } \\
\text { private centres. }\end{array}$ & $\begin{array}{l}\text { Women who } \\
\text { request it }\end{array}$ & $\begin{array}{c}\text { Ad hoc } \\
\text { Questionnaire }\end{array}$ & $\begin{array}{c}\text { 4,390 } \\
\text { women }\end{array}$ & - \\
\hline 2005 & $\begin{array}{c}\text { González A } \\
\text { (17) }\end{array}$ & Madrid & $2002-2004$ & $\begin{array}{c}\text { Primary care } \\
\text { centres }\end{array}$ & $\begin{array}{l}\text { Women who } \\
\text { request it }\end{array}$ & Clinical histories & $\begin{array}{c}286 \\
\text { women }\end{array}$ & $14-44$ \\
\hline 2005 & Torres C (18) & Lleida & $1999-2001$ & $\begin{array}{c}\text { Family planning } \\
\text { centres }\end{array}$ & $\begin{array}{l}\text { Women who } \\
\text { request it }\end{array}$ & $\begin{array}{c}\text { Ad hoc } \\
\text { Questionnaire }\end{array}$ & $\begin{array}{c}2,813 \\
\text { women }\end{array}$ & E13-53 \\
\hline 2005 & $\begin{array}{c}\text { Martínez- } \\
\text { Zamora MA } \\
(19)\end{array}$ & Barcelona & 2002 & $\begin{array}{l}\text { Gynaecology } \\
\text { Hospital } \\
\text { emergency } \\
\text { departments }\end{array}$ & $\begin{array}{l}\text { Women who } \\
\text { request it }\end{array}$ & $\begin{array}{c}\text { Ad hoc } \\
\text { Questionnaire }\end{array}$ & $\begin{array}{c}400 \\
\text { women }\end{array}$ & $14-44$ \\
\hline 2006 & $\begin{array}{c}\text { Santamaría } \\
\text { T (20) }\end{array}$ & Toledo & $2002-2004$ & $\begin{array}{l}\text { Primary care } \\
\text { emergency } \\
\text { departments }\end{array}$ & $\begin{array}{l}\text { Women who } \\
\text { request it }\end{array}$ & $\begin{array}{c}\text { Ad hoc } \\
\text { Questionnaire }\end{array}$ & $\begin{array}{c}132 \\
\text { women }\end{array}$ & $14-46$ \\
\hline 2006 & $\begin{array}{c}\text { González- } \\
\text { Mohino MB } \\
(21)\end{array}$ & Lanzarote & 2003-2004 & $\begin{array}{c}\text { Hospital } \\
\text { emergency } \\
\text { rooms and } \\
\text { primary care } \\
\text { emergency } \\
\text { departments }\end{array}$ & $\begin{array}{l}\text { Women who } \\
\text { request it }\end{array}$ & $\begin{array}{c}\text { Ad hoc } \\
\text { Questionnaire }\end{array}$ & $\begin{array}{c}52 \\
\text { women }\end{array}$ & $15-37$ \\
\hline 2007 & $\begin{array}{l}\text { Luengo P } \\
\text { (22) }\end{array}$ & Alicante & 2005 & $\begin{array}{l}\text { First-year } \\
\text { and third year } \\
\text { students of } \\
\text { Nutrition and } \\
\text { Economic }\end{array}$ & $\begin{array}{l}\text { College } \\
\text { students }\end{array}$ & $\begin{array}{c}\text { Ad hoc } \\
\text { Questionnaire }\end{array}$ & $\begin{array}{l}184 \text { men } \\
\text { and } \\
\text { women }\end{array}$ & - \\
\hline 2008 & $\begin{array}{l}\text { Sarrat MA } \\
\quad(23)\end{array}$ & Zaragoza & $2005-2006$ & Hospital & $\begin{array}{l}\text { Women who } \\
\text { request it }\end{array}$ & Clinical histories & $\begin{array}{c}1,007 \\
\text { women }\end{array}$ & $15-43$ \\
\hline 2008 & $\begin{array}{c}\text { Fuentes MD } \\
(24)\end{array}$ & Ourense & $2006-2007$ & $\begin{array}{l}\text { Primary care } \\
\text { emergency } \\
\text { departments }\end{array}$ & $\begin{array}{l}\text { Women who } \\
\text { request it }\end{array}$ & $\begin{array}{c}\text { Ad hoc } \\
\text { Questionnaire }\end{array}$ & $\begin{array}{c}188 \\
\text { women }\end{array}$ & $14-51$ \\
\hline 2009 & Ros C (1) & Catalonia & 2004-2007 & $\begin{array}{l}\text { Data added } \\
\text { maternal and } \\
\text { child health } \\
\text { Programme, } \\
\text { Department of } \\
\text { Public Health }\end{array}$ & $\begin{array}{l}\text { Women who } \\
\text { request it }\end{array}$ & $\begin{array}{l}\text { Listing data } \\
\text { including } \\
\text { minimum }\end{array}$ & $\begin{array}{l}17,149 \\
\text { women }\end{array}$ & $13-49$ \\
\hline 2010 & $\begin{array}{l}\text { López- } \\
\text { Amorós M } \\
\text { (25) }\end{array}$ & Terrassa & 2008 & Schools & Adolescents & $\begin{array}{c}\text { Study monitoring } \\
\text { health } \\
\text { behaviours }\end{array}$ & $\begin{array}{l}390 \text { men } \\
\text { and } \\
\text { women }\end{array}$ & $14-18$ \\
\hline
\end{tabular}

Figure 1 - Selected articles on the use of the morning after pill in the Spanish population. Palma, Illes Balears, Spain, 2011 
Figure 2 shows the socio-demographic variables of the study subjects. In twelve of the fourteen articles the average age of the women who sought EC was reported and found to be between 21 and 24 years. In only half of the studies (7) information was provided on educational level showing that, among women requesting EC, university students accounted for between $19.7 \%$ and $52.6 \%$. Only two studies collected data on civil status and these showed a high percentage of single women. Regarding employment, in the five studies that included this variable, the largest percentage of the study population was students.

\begin{tabular}{|c|c|c|c|c|c|c|c|c|c|c|c|c|}
\hline \multirow{2}{*}{$\begin{array}{c}\text { Year of } \\
\text { publica- } \\
\text { tion }\end{array}$} & \multirow[b]{2}{*}{$\begin{array}{l}\text { First } \\
\text { author }\end{array}$} & \multicolumn{3}{|c|}{ Age } & \multicolumn{3}{|c|}{ Marital status } & \multicolumn{3}{|c|}{ Educational level } & \multicolumn{2}{|c|}{$\begin{array}{l}\text { Employment } \\
\text { status }\end{array}$} \\
\hline & & Average & $\begin{array}{c}\text { Mi- } \\
\text { nors } \\
(\%)\end{array}$ & Others & $\begin{array}{l}\text { Sin- } \\
\text { gle } \\
(\%)\end{array}$ & $\begin{array}{c}\text { Mar- } \\
\text { ried } \\
(\%)\end{array}$ & $\begin{array}{c}\text { Sepa- } \\
\text { rated } \\
(\%)\end{array}$ & $\begin{array}{c}\text { Higher } \\
\text { educa- } \\
\text { tion } \\
(\%)\end{array}$ & $\begin{array}{l}\text { Sec- } \\
\text { ondary } \\
\text { educa- } \\
\text { tion (\%) }\end{array}$ & $\begin{array}{c}\text { Primary } \\
\text { educa- } \\
\text { tion } \\
(\%)\end{array}$ & $\begin{array}{c}\text { Stu- } \\
\text { dents } \\
(\%)\end{array}$ & $\begin{array}{l}\text { Work- } \\
\text { ers } \\
(\%)\end{array}$ \\
\hline 2002 & $\begin{array}{l}\text { Aginaga } \\
\text { JR (11) }\end{array}$ & 22 & * & * & * & * & * & 52.6 & 28.1 & 19.4 & 51.3 & 42 \\
\hline 2002 & $\begin{array}{l}\text { Cárdenas } \\
\text { DP (12) }\end{array}$ & $21.3 \pm 5.4$ & * & $\begin{array}{c}87 \% \\
<30 \text { years }\end{array}$ & * & * & * & * & * & * & * & * \\
\hline 2002 & Ruíz S (13) & 23.9 & 9.9 & * & * & * & * & 40.9 & 33.9 & 4.6 & * & * \\
\hline 2003 & Lete I (14) & 23 & 1.9 & $\begin{array}{c}37 \% \\
<19 \text { years } \\
71 \% \\
<24 \text { years }\end{array}$ & * & * & * & 19.7 & * & 1.1 & 40 & 31.8 \\
\hline 2005 & $\begin{array}{l}\text { González } \\
\text { A (15) }\end{array}$ & 32.6 & * & $\begin{array}{c}23 \% \\
<20 \text { years }\end{array}$ & * & * & * & * & * & * & * & * \\
\hline 2005 & $\begin{array}{c}\text { Torres C } \\
(16)\end{array}$ & 21.5 & * & * & * & * & * & 33.9 & 39.1 & 1.7 & * & * \\
\hline 2005 & $\begin{array}{l}\text { Martínez- } \\
\text { Zamora } \\
\text { MA (17) }\end{array}$ & 23 & 6.75 & * & * & * & * & 22 & 61 & 17 & 54 & 37 \\
\hline 2006 & $\begin{array}{l}\text { Santama- } \\
\text { ría T (18) }\end{array}$ & 22.9 & * & * & 85.6 & 12.1 & 2.3 & * & * & * & 45.8 & * \\
\hline 2006 & $\begin{array}{l}\text { González- } \\
\text { Mohino MB } \\
\text { (19) }\end{array}$ & 24.2 & * & * & * & * & * & * & * & 44.2 & * & * \\
\hline 2007 & $\begin{array}{l}\text { Luengo P } \\
(20)\end{array}$ & 21.59 & * & * & 93.4 & * & * & 100 & * & * & 100 & * \\
\hline 2008 & $\begin{array}{c}\text { Sarrat MA } \\
(21)\end{array}$ & 21 & 7.3 & * & * & * & * & * & * & * & * & * \\
\hline 2008 & $\begin{array}{l}\text { Fuentes } \\
\text { MD (22) }\end{array}$ & 23.3 & 25.3 & * & * & * & * & * & * & * & * & * \\
\hline 2009 & Ros C (23) & * & * & $16-19$ & * & * & * & * & * & * & * & * \\
\hline 2010 & $\begin{array}{c}\text { López- } \\
\text { Amorós M } \\
(24)\end{array}$ & * & * & * & * & * & * & * & * & * & * & * \\
\hline
\end{tabular}

Figure 2 - Distribution of socio-economic variables of women using emergency contraception, according to age, marital status, educational level and employment status. Palma, Illes Balears, Spain, 2011 
With respect to the use profile (Figure 3), the ten studies that included this variable reported that the majority of women stated that they had used EC only once. It should be noted that between $9-60 \%$ of women had used it on more than one occasion. In a study ${ }^{(22)}$, $58.8 \%$ of repeated users were under 20 years of age. Time elapsed since unprotected coitus was recorded in eight studies and it appears that a very high percentage of women seek EC within 48 hours. The reason given by these women for requesting and using EC was reported in ten studies and mainly referred to condom breakage. In another study ${ }^{(11)}, 27.6 \%$ stated they used EC as a contraceptive method. Finally, the weekends were the time of greatest demand, followed by Mondays. It is noteworthy that some $7 \%$ of women interviewed in one study ${ }^{(13)}$ continued to engage in unprotected coitus during the same menstrual cycle after taking EC.

\begin{tabular}{|c|c|c|c|c|c|c|c|c|c|c|}
\hline \multirow{2}{*}{$\begin{array}{l}\text { Year of } \\
\text { public- } \\
\text { ation }\end{array}$} & \multirow{2}{*}{$\begin{array}{l}\text { First } \\
\text { author }\end{array}$} & \multicolumn{2}{|c|}{ Use } & \multicolumn{3}{|c|}{$\begin{array}{l}\text { Elapsed time after unprotected } \\
\text { coitus }\end{array}$} & \multicolumn{2}{|c|}{ Reason asserted } & \multicolumn{2}{|c|}{$\begin{array}{l}\text { Days of week } \\
\text { demanded }\end{array}$} \\
\hline & & $\begin{array}{c}\text { Once } \\
(\%)\end{array}$ & $\begin{array}{c}\text { More } \\
\text { than } \\
\text { once } \\
(\%)\end{array}$ & $\begin{array}{l}\text { Average } \\
\text { in hours }\end{array}$ & $\begin{array}{c}<24 \mathrm{~h}-48 \mathrm{~h} \\
(\%)\end{array}$ & $\begin{array}{l}\text { 48h-72h } \\
(\%)\end{array}$ & $\begin{array}{c}\text { Condom } \\
\text { breakage } \\
(\%)\end{array}$ & $\begin{array}{c}\text { No } \\
\text { Contraceptive } \\
(\%)\end{array}$ & $\begin{array}{l}\text { Weekend } \\
(\%)\end{array}$ & $\begin{array}{c}\text { Monday } \\
(\%)\end{array}$ \\
\hline 2002 & $\begin{array}{l}\text { Aginaga Jr } \\
\text { (11) }\end{array}$ & * & * & * & 98.7 & * & 67.5 & 8.8 & * & * \\
\hline 2002 & $\begin{array}{l}\text { Cárdenas } \\
\text { Dp (12) }\end{array}$ & 88 & 12 & * & 96 & 4 & 83 & 15 & * & * \\
\hline 2002 & Ruíz S (13) & * & 19.5 & 31.5 & * & * & 69.3 & 11.5 & * & 47.4 \\
\hline 2003 & Lete I (14) & 80.2 & 19.8 & * & 91.5 & 5.6 & 68.7 & 15.4 & * & 28.6 \\
\hline 2005 & $\begin{array}{l}\text { González A } \\
\text { (15) }\end{array}$ & * & * & * & * & * & * & * & * & * \\
\hline 2005 & $\begin{array}{c}\text { Torres C } \\
(16)\end{array}$ & 72.3 & * & 33.7 & * & * & 62.1 & * & * & * \\
\hline 2005 & $\begin{array}{l}\text { Martínez- } \\
\text { Zamora Ma } \\
\quad(17)\end{array}$ & 64.26 & 35.7 & 15 & * & * & * & * & * & * \\
\hline 2006 & $\begin{array}{l}\text { Santamaría } \\
\quad \mathrm{T}(18)\end{array}$ & * & 24.4 & 14.5 & * & * & 75.8 & 17.7 & * & * \\
\hline 2006 & $\begin{array}{l}\text { González- } \\
\text { Mohino Mb } \\
\text { (19) }\end{array}$ & * & 26.9 & * & * & * & 73.1 & * & 65.4 & * \\
\hline 2007 & $\begin{array}{l}\text { Luengo P } \\
\quad(20)\end{array}$ & * & * & * & * & * & 78.4 & * & * & * \\
\hline 2008 & $\begin{array}{l}\text { Sarrat Ma } \\
\quad(21)\end{array}$ & * & 60 & * & 78 & 12 & 79.3 & 7 & 52 & 12 \\
\hline 2008 & $\begin{array}{l}\text { Fuentes Md } \\
\quad(22)\end{array}$ & 61.6 & 38.4 & * & * & * & 78.6 & 18.6 & 48.4 & 16.1 \\
\hline 2009 & Ros C (23) & 84.2 & 9 & * & * & * & * & * & * & * \\
\hline 2010 & $\begin{array}{l}\text { López- } \\
\text { Amorós M } \\
(24)\end{array}$ & 53 & 33 & * & * & * & * & * & * & * \\
\hline
\end{tabular}

Figure 3 - Variables distribution using EC, according to use, elapsed time after unprotected coitus, reason asserted and days of week demanded. Palma, Illes Balears, Spain, 2011 
Sexual habits and habitual contraceptive method are shown in Figure 4. Data on the usual method of contraception were recorded in nine studies. All of these demonstrated that among EC users condoms were most frequently used, followed at some considerable distance, by oral contraception. Between $3 \%$ and
$19 \%$ of the interviewees did not use any contraceptive method. Regarding the age at initiation of penetrative sexual relations, data were only gathered in three articles and was found to be between 16.9 and 18 years. Obstetrics history was recorded in few studies, reporting that between 6.1 and $9.5 \%$ of women surveyed stated having had an abortion on at least one occasion.

\begin{tabular}{|c|c|c|c|c|c|c|c|c|}
\hline \multirow{2}{*}{$\begin{array}{l}\text { Year of } \\
\text { publication }\end{array}$} & \multirow{2}{*}{ First author } & \multicolumn{3}{|c|}{ Usual contraception } & \multicolumn{2}{|c|}{$\begin{array}{l}\text { Initiation of sexual } \\
\text { relations }\end{array}$} & \multicolumn{2}{|c|}{ Obstetrics history } \\
\hline & & $\begin{array}{l}\text { Condom } \\
(\%)\end{array}$ & $\begin{array}{c}\text { Oral } \\
\text { contraception (\%) }\end{array}$ & $\begin{array}{l}\text { None } \\
(\%)\end{array}$ & $\begin{array}{l}\text { Average } \\
\text { age }\end{array}$ & Range & $\begin{array}{l}\text { Children/ } \\
\text { pregnancy }\end{array}$ & Abortions \\
\hline 2002 & $\begin{array}{l}\text { Aginaga Jr } \\
\quad(11)\end{array}$ & * & * & * & * & * & 9.9 & 7.3 \\
\hline 2002 & $\begin{array}{c}\text { Cárdenas } \mathrm{Dp} \\
\text { (12) }\end{array}$ & 85 & * & 17 & * & * & * & * \\
\hline 2002 & Ruíz S (13) & 94.5 & * & 3.1 & * & * & * & 6.5 \\
\hline 2003 & Lete I (14) & 82.2 & 5.8 & 8.8 & * & * & * & * \\
\hline 2005 & $\begin{array}{c}\text { González A } \\
\text { (15) }\end{array}$ & * & * & * & * & * & * & * \\
\hline 2005 & Torres C (16) & 73.2 & * & * & 17.8 & 9-32 & * & * \\
\hline 2005 & $\begin{array}{c}\text { Martínez- } \\
\text { Zamora Ma } \\
\quad(17)\end{array}$ & * & * & * & 18 & $13-30$ & * & 9.5 \\
\hline 2006 & $\begin{array}{l}\text { Santamaría } \\
\mathrm{T}(18)\end{array}$ & 80.2 & 7.6 & 12.2 & * & * & 20.5 & 6.1 \\
\hline 2006 & $\begin{array}{l}\text { González- } \\
\text { Mohino Mb } \\
\text { (19) }\end{array}$ & * & * & 19.2 & * & * & * & * \\
\hline 2007 & Luengo P (20) & 97.1 & * & * & 16.9 & * & * & * \\
\hline 2008 & Sarrat Ma (21) & 77 & * & 10 & * & * & * & * \\
\hline 2008 & $\begin{array}{l}\text { Fuentes Md } \\
\text { (22) }\end{array}$ & * & * & 17 & * & * & * & * \\
\hline 2009 & Ros C (23) & * & * & * & * & * & * & * \\
\hline 2010 & $\begin{array}{l}\text { López-Amorós } \\
\text { M (24) }\end{array}$ & * & * & * & * & * & * & * \\
\hline
\end{tabular}

Figure 4 - Sexual behaviour of women using emergency contraception, according to usual contraception, initiation of sexual relations and obstetrics history. Palma, Illes Balears, Spain, 2011 


\section{Discussion}

Based on these data, we discuss the effectiveness of the dispensation of this drug in Family Planning Centres as well as the inclusion of cases from these centres and those based on Clinical Histories.

There are Spanish regions where the prescription was free and others in which the drug was paid. As it was free in Andalucía, the article made in this region (12) should be analysed in a different manner.

Our study shows that, in general, the studies analysed present limited results that do not allow a clear, detailed picture to emerge of the characteristics of women using EC. Study results show great variability reflecting the various contexts in which the studies have been performed and cover primary care emergency departments, hospital emergency rooms, family planning centres, the general population, the university population, and secondary school students. This could explain, in turn, the considerable variability in the estimated parameters. The sample size in the published and selected studies used for analysis is also highly diverse, ranging from 52 up to 17,149 women. Moreover, it can be seen that the number of variables studied is limited and varies between studies, hampering attempts to view the profile of users in depth. For instance, studies performed outside Spain report on the use of other drugs among EC users and observe an association between consumption of alcohol and tobacco, number of sexual partners and use of EC, while in the reviewed studies these variables were not investigated (25-28). Nor was any study found where comparisons were made with women who had not taken EC, which would have allowed clarification of the characteristics that differentiate these women and the factors related to the use of EC. Similarly, in the United States and the United Kingdom, some qualitative studies have been carried out which provide an insight into the discourse of women on the free dispensation of EC in pharmacies or advance supply of 5 units of the drug (29-32). This research suggests that free dispensation increases access to EC that is highly valued by the women as it reduces waiting times, thus increasing efficacy as the drug is always administered within 72 hours of unprotected coitus. However, we did not find any qualitative studies performed in Spain, which further hinders the collection of detailed information on the views and experiences of women who use or are thinking of using EC. The availability of this type of study would also allow identification of relevant variables that could be used in quantitative studies. Finally, the variables considered in the articles obtained have been collected in a disparate manner making comparison difficult.

This study contributes to the advancement of scientific knowledge with knowledge of the actual profile of users of emergency contraception in Spain. This will allow us to program affective-sexual health strategies adjusted to the reality, at all levels: preventive, promotion, health education and health care; and reorient the accessibility of gynaecological services.

\section{Conclusion}

A major limitation found when performing the systematic review is that there are few studies published in the literature on emergency contraception in Spain and almost all of them are descriptive. The lack of homogeneity and comprehensiveness of the variables gathered only allows for a limited view of the profile of EC users and these individuals need to be studied in greater depth if educational interventions are to be designed within the framework of sexual health for potential users. In addition, studies are also urgently required comparing women who use EC with those who do not. Despite these drawbacks, using those variables that have data collected by the majority of articles, it can be observed that the profile of the EC user is a young, single woman who attends emergency services at the weekends, within 48 hours of unprotected coitus. Further study is required to understand the impact of free dispensation of EC, the profile of users and nonusers, and their habits with respect to emergency contraception.

\section{References}

1. Merino Merino B, Lizarbe Alonso VM, Koerting de Castro A, Diezma Criado JC, Delicado Gálvez I, Echeverría Cubillas $P$, et al. Ganar salud con la juventud: Nuevas Recomendaciones sobre Salud Sexual y Reproductiva, consumo de Alcohol y Salud Mental, del Grupo de Trabajo de Promoción de la Salud a la Comisión de Salud Pública del Consejo Interterritorial del Sistema Nacional de Salud [Internet]. Madrid: Ministerio de Sanidad y Consumo, Instituto de la Juventud; 2008 [Acess: $13 \mathrm{fev}$ 2006] Available from: http://www.msssi.gob.es/ciudadanos/ proteccionSalud/adolescencia/docs/jovenes_2008.pdf.

2. Valandia MA. Estrategias docentes de Enfermería para el abordaje de la sexualidad, la salud sexual y la salud reproductiva en adolescentes y jóvenes [tesis doctoral]. Alacant: Departamento de Enfermería, Universidad d'Alacant; 2011 
3. Delgado M, Barrios L. El aborto en España en una perspectiva internacional. Est Geográficos. 2005;66(258) doi:10.3989/egeogr.2005.i258.156.

4. Ministerio de Sanidad, Política Social e Igualdad (ES). Interrupción Voluntaria del Embarazo. Datos definitivos correspondientes al año 2009. Madrid; 2010.

5. Acosta Navas B, Muñoz Hiraldo ME. Contracepción postcoital de urgencia. Rev Ped de Aten Primaria. 2006;8(31):147-154. ni4.

6. Polis CB, Grimes DA, Schaffer K, Blanchard K, Glasier A, Harper C. Advance provision of emergency contraception for pregnancy prevention. Cochrane Database of Systematic Rev. 2007; Issue 2. Art. No.: CD005497. DOI: 10.1002/14651858.CD005497.pub2.

7. Glasier A. Emergency contraception. In: Millar RP, Baird DY, editors. Human Reproduction: pharmaceutical and technical advances. Br Med Bull. 56(3):729-38.

8. Martínez-González MA, De Irala J. Medicina Preventiva y fracaso clamoroso de la salud pública: llegamos mal porque llegamos tarde. Med Clin. (Barc). 2005;124(17):656-60.

9. Glasier A, Fairhurst K, Wyke S, Ziebland S, Reaman $P$, Walter $J$, et al. Advanced provision of emergency contraception does not reduce abortion rates. Contraception. 2004;69:361-6.

10. Bissell $P$, Anderson C. Supplying emergency contraception via community pharmacies in the UK: reflections on the experiences of users and providers. Soc Sci Med. 2003;57(12):2367-78.

11. Aginaga Badiola JR, González Santo Tomás R, Guillem Martínez E, Cerdiera Benito C, Reguera Cámara A, Madrid Balbas A. Análisis de las demandantes de pastillas anticonceptivas de urgencia. Emergencias. 2002; 14:125-9.

12. Cárdenas Cruz DP, Parrilla Ruiz F, Mengíbar Gómez MM, Espinosa Fernández M, Issa Khozouz Ziad, Cárdenas Cruz A. Perfil de la solicitante de la píldora postcoital (Levonorgestrel) en unidades de urgencias. MEDIFAM. 2002;3(3):179-83.

13. Ruiz S, Güell E, Herranz C, Pedraza C. Anticoncepción poscoital. Características de la demanda. Aten Primaria. 2002;30(6):381-7.

14. Lete I, Cabero L, Álvarez D, Ollé C. Observational study on the use of emergency contraception in Spain: results of a national survey. Eur J Contracept Reprod Heallth Care. 2003;8:203-9.

15. González A, Éngel JL. Los médicos varones reciben menos solicitudes de anticoncepción de emergencia que sus colegas mujeres. Aten Primaria. 2005;35(9):493.

16. Torres $C$, Vilaplana $D$, Sáez $S$, Pérez R, Martínez M. Intercepción poscoital hormonal en un servicio de planificación familiar. Enferm Clín. 2005;15(5):257-61.
17. Martínez-Zamora MA, Bellart J. Anticoncepción poscoital con levonorgestrel: estudio sociodemográfico. Med Clin. (Barc). 2005;125(2):75-9.

18. Santamaría Rodríguez T, Crespo del Pozo AV, Cid Prados A, Gontán García-Salamanca MJ, González Pérez M, Baz Collado C, et al. Anticoncepción de emergencia: perfil de las usuarias y características de la demanda. SEMERGEN. 2006;32:321-4.

19. González-Mohino Loro MB, Cáceres Montero E, Anduaga Aguirre MA, Blanco López B, Regueira Martínez AM, Romero Brufao S. Demandantes de las pastillas anticonceptivas de urgencia en Lanzarote. Análisis descriptivo. SEMERGEN. 2006;81:840-842.

20. Luengo P, Orts I, Caparrós RA, Arroyo OI. Comportamiento sexual, prácticas de riesgo y anticoncepción en jóvenes universitarios de Alicante. Enferm Clín. 2007;17(2):85-9.

21. Sarrat MA, Yáñez F, Vicente A, Muñoz S, Alcalá J, Royo R. Anticoncepción de emergencia en un servicio de urgencias hospitalario: la experiencia de un año de gratuidad. Emergencias. 2008;20:108-12.

22. Fuentes Lema MD, López Pérez A, Alonso Pequeño MM. Demanda de anticoncepción de emergencia en el punto de atención continuada de Verín. Cuad de Aten Primaria. 2008; 15:189-92.

23. Ros C, Miret M, Rué M. Estudio descriptivo sobre el uso de la anticoncepción de emergencia en Cataluña. Comparación entre una zona rural y una urbana. Gac Sanit. 2009;23(6):496-500.

24. López-Amorós M, Schiaffino A, Moncada A, Pérez G. Factores asociados al uso autodeclarado de la anticoncepción de emergencia en la población escolarizada de 14 a 18 años de edad. Gac Sanit. 2010;24(5):404-9.

25. Loughrey F, Matthews A, Bedford D, Howell F. Characteristics of women seeking emergency contraception in General Practice. IMJ. 2006:99:50-2.

26. Black $\mathrm{KI}$, Mercer $\mathrm{CH}$, Johnson AM, Wellings $\mathrm{K}$. Sociodemographic and sexual health profile of users of emergency hormonal contraception: data from a British probability sample survey. Contraception. 2006;74:30912.

27. Goulard H, Monreau C, Gilbert F, Job-Spira N, Bajos $\mathrm{N}$; Cocon Group. Contraceptive failures and determinats of emergency contraception use. Contraception. 2006; 74:208-13.

28. Falah-Hassany K, Emergency contraception among Finnish adolescents: awareness, use and the effect of non-prescription status. BMC Public Health. 2007; 7:201. 29. Ziebland S, Wyke S, Reaman P, Fairhurst K, Walter J, Glasier A. What happened when Scottisch women were given advance supplies of emergency contraception? 
A survey and qualitative study of women's views and experiences. Soc Scien Med. 2005;60 1767-79.

30. Hayes M, Hutchings J, Hayes P. Reducing Unintended Pregnancy by Increasing Acces to Emergency Contraceptive Pills. Maternal Child Health J. 2000;4(3):203-8.

31. Fairhurst K, Ziebland S, Wyke S, Reaman P, Glasier A. Emergency contraception: why can't you give it away? Qualitative findings from an evaluation advance provision of emergency contraception. Contraception. 2004;70:25-9.

32. Karasz A, Kirchen NT, Gold M. The visit before the morning after: barriers to preprescribing emergency contraception. Ann Fam Med. 2004;2(4):345-50.

Copyright $\odot 2016$ Revista Latino-Americana de Enfermagem This is an Open Access article distributed under the terms of the Creative Commons (CC BY).

This license lets others distribute, remix, tweak, and build upon your work, even commercially, as long as they credit you for the original creation. This is the most accommodating of licenses offered. Recommended for maximum dissemination and use of licensed materials. 